\title{
Universal power law of the gravity wave manifestation in the AIM CIPS polar mesospheric cloud images
}

\author{
Pingping Rong ${ }^{1}$, Jia Yue ${ }^{1,2}$, James M. Russell III ${ }^{1}$, David E. Siskind ${ }^{3}$, and Cora E. Randall ${ }^{4,5}$ \\ ${ }^{1}$ Center for Atmospheric Sciences, Hampton University, Hampton, VA 23668, USA \\ ${ }^{2}$ Earth System Science Interdisciplinary Center, University of Maryland, College Park, MD 20740, USA \\ ${ }^{3}$ Space Science Division, Naval Research Laboratory, Washington D.C., WA 20375, USA \\ ${ }^{4}$ Laboratory for Atmospheric and Space Physics, University of Colorado Boulder, Boulder, CO 80303, USA \\ ${ }^{5}$ Department of Atmospheric and Oceanic Sciences, University of Colorado Boulder, Boulder, CO 80309, USA
}

Correspondence: Pingping Rong (ping-ping.rong@ @amptonu.edu)

Received: 4 August 2017 - Discussion started: 16 August 2017

Revised: 26 November 2017 - Accepted: 16 December 2017 - Published: 24 January 2018

\begin{abstract}
We aim to extract a universal law that governs the gravity wave manifestation in polar mesospheric clouds (PMCs). Gravity wave morphology and the clarity level of display vary throughout the wave population manifested by the PMC albedo data. Higher clarity refers to more distinct exhibition of the features, which often correspond to larger variances and a better-organized nature. A gravity wave tracking algorithm based on the continuous Morlet wavelet transform is applied to the PMC albedo data at $83 \mathrm{~km}$ altitude taken by the Aeronomy of Ice in the Mesosphere (AIM) Cloud Imaging and Particle Size (CIPS) instrument to obtain a large ensemble of the gravity wave detections. The horizontal wavelengths in the range of $\sim 20-60 \mathrm{~km}$ are the focus of the study. It shows that the albedo (wave) power statistically increases as the background gets brighter. We resample the wave detections to conform to a normal distribution to examine the wave morphology and display clarity beyond the cloud brightness impact. Sample cases are selected at the two tails and the peak of the normal distribution to represent the full set of wave detections. For these cases the albedo power spectra follow exponential decay toward smaller scales. The high-albedo-power category has the most rapid decay (i.e., exponent $=-3.2$ ) and corresponds to the most distinct wave display. The wave display becomes increasingly blurrier for the medium- and low-power categories, which hold the monotonically decreasing spectral exponents of -2.9 and -2.5 , respectively. The majority of waves are straight waves whose clarity levels can collapse between the different brightness levels, but in the brighter back-
\end{abstract}

ground the wave signatures seem to exhibit mildly turbulentlike behavior.

\section{Introduction}

Atmospheric gravity waves play important roles in atmospheric circulation, structure, and variability. The influence of breaking gravity waves on the dynamics and chemical composition of the $60-110 \mathrm{~km}$ region has been the most significant. The momentum deposited by breaking waves at mesospheric altitudes reverses the zonal winds, drives a strong mean meridional circulation, and produces a very cold polar summer mesopause region that enables polar mesospheric clouds (PMCs) to form (Fritts and Alexander, 2003; Garcia and Solomon, 1985). Aside from causing these indirect but fundamental effects on global circulation, gravity waves also have widespread displays in PMCs (e.g., Fogle and Haurwitz, 1966; Fritts et al., 1993; Dalin et al., 2010; Taylor et al., 2011; Thurairajah et al., 2013; Yue et al., 2014), serving as a visible manifestation of the polar summer mesospheric dynamics.

Semi-organized wave-like structures have been the most characteristic and widespread features in PMCs. PMCs are also referred to as noctilucent clouds (NLCs) when observed from the ground. In an extensive review given by Fogle and Haurwitz (1966), four types of NLCs are categorized at a descriptive level: bands and long streaks, billows, whirls, and veils. Most of these features resembled semi-organized 
wave signatures. These NLC features are reflections of gravity waves, gravity wave breaking, or wave-breaking-induced turbulence (e.g., Fritts et al., 1993; Dalin et al., 2010; Baumgarten and Fritts, 2014; Miller et al., 2015). Gravity wave horizontal scales encompass an extremely broad range of $\sim 10-1000 \mathrm{~km}$ (e.g., Fritts and Alexander, 2003), but the most widespread and readily observed displays in the NLCs are at wavelengths shorter than $100 \mathrm{~km}$ (e.g., Fogle and Haurwitz, 1966). Near-infrared hydroxyl (OH) airglow images have also revealed similar wave patterns. For example, Taylor and Edwards (1991) observed several 15-20 km wavelength linear wave patterns over Hawaii in March, and Yue et al. (2009) reported capturing the mesospheric concentric waves with wavelengths in the range of $\sim 40-80 \mathrm{~km}$ over Colorado and a few neighboring states.

The Aeronomy of Ice in the Mesosphere (AIM) satellite was launched in April 2007, becoming the first satellite mission dedicated to the study of PMCs (Russell III et al., 2009). One of the primary research goals of the AIM mission is to explore how PMCs form and vary. In pursuing this goal, gravity waves have become an increasingly important topic in AIM science investigations. The Cloud Imaging and Particle Size (CIPS) instrument (McClintock et al., 2009) aboard the AIM satellite provides PMC images that cover the polar region daily throughout the summer season in both hemispheres, and it has collected almost 10 years of data to date. These data have enabled extensive studies of gravity wave signatures in PMCs and of mesospheric dynamics more generally (e.g., Thurairajah et al., 2013; Yue et al., 2014). Thurairajah et al. (2013) presented a host of characteristic cloud structures in the CIPS PMC images, among which the noteworthy ones include the "void" feature with a clean edge and a core region of sharply reduced cloud brightness, and concentric waves (see also Taylor et al., 2011; Yue et al., 2014). These are fairly unique signatures with low occurrence frequency and are not the focus of the current study, although we do also discuss some examples of the concentric waves in a later part of this paper. Yue et al. (2014) correlated concentric wave patterns in the CIPS PMC data with similar patterns in the stratosphere observed by the Atmospheric Infrared Sounder (AIRS) instrument aboard NASA's Aqua satellite (Aumann et al., 2003). Concentric waves are the most evident proof that gravity waves excited by tropospheric storm systems have propagated into the mesosphere.

Quantitatively characterizing gravity waves in PMC images is generally difficult because the wave patterns are complex, although several NLC morphology types have been successfully interpreted in previous modeling studies (e.g., Fritts et al., 1993; Baumgarten and Fritts, 2014). In addition, PMCs are characterized by a hierarchy of larger-scale features $(\sim 100-1000 \mathrm{~km})$ that often obscure the smallerscale $(<100 \mathrm{~km})$ gravity wave signatures. It is worth noting that these large-scale features may not be exclusively gravity wave structures because tides and planetary waves also play important roles in modulating the PMC spatial variability (e.g., Merkel et al., 2009).

In this study we developed an algorithm to quantify the occurrence and manifestation of gravity waves with horizontal wavelengths of $\sim 20-60 \mathrm{~km}$ in the CIPS Level 2 albedo data (Lumpe et al., 2013). Such a scale range is chosen because the full display of these waves can fit well into one CIPS orbital strip and also because these short-wavelength waves are proven to be the most commonly observed. We aim at obtaining a universal law that governs the wave display for a large ensemble of semi-organized wave structures via sorting their albedo disturbance power and examining their relationship with the background cloud brightness. The law obtained will go beyond the apparent dependence of the albedo wave power on the background cloud brightness because the real effect of the gravity waves on the PMC brightness is through dynamical or microphysical control, which is related to the variability in winds, temperature, and water vapor, as is shown in the model studies carried out by Jensen and Thomas (1994) and Chandran et al. (2012) for instance. These studies suggested that both long and short gravity waves eventually reduce the cloud brightness locally. Generally speaking, the fundamental relationship between the gravity waves and the PMC brightness is not yet fully understood. In this study we do not pursue this fundamental relationship or attempt to characterize specific wave events in a strict sense.

The algorithm we designed for CIPS differs from the wave tracking approaches proposed in some other research papers (e.g., Chandran et al., 2010; Gong et al., 2015) in the sense that it confines the scale range first, rather than searching for spectral peaks. This is because the wave patterns in PMCs can be obscured by larger-scale variability that possesses larger amplitudes and also because these patterns are rarely monochromatic. As a result, spectral peaks, for example in wave numbers, do not stand out easily unless a distinct wave structure is first visually detected and then a spectral analysis is carried out along its most optimum orientation. Such a challenge is also reflected in the airglow image processing that aims at identifying the mesospheric gravity waves (e.g., Matsuda et al., 2014). In addition, it is worth mentioning that in the current study we did not adopt high-pass filtering to extract the small-scale structures (e.g., Chandran et al., 2010) because 2-dimensional (2-D) filtering is prone to inducing notable artificial features if large and small scales are not separated optimally. Another important technique to detect gravity waves and to resolve their characteristics is applying a spatiotemporal analysis to either the ground-based or satellite measurements (e.g., Wachter et al., 2015; Ern et al., 2011). For example, Wachter et al. (2015) applied such a technique to the $\mathrm{OH}$ airglow time series measured at the chosen triangular equilateral ground sites to yield a consistent set of wave parameters. In these analyses, however, a full display of the waves is not captured because only a few locations are used. CIPS, on the other hand, has extended spatial coverage, 
and therefore in the current study we aim to only detect the spatial wave patterns.

The structure of the paper is as follows. A brief description of the CIPS data is given in Sect. 2. In Sect. 3 we describe the wave tracking approach, provide an analytical demonstration, and then apply the algorithm to a few concentric wave patterns found in the CIPS imagery. In Sect. 4 the statistics of the wave power are obtained, and their dependence on the background cloud brightness is quantified. A resampling process is applied to reach a normal distribution of all the wave power values. Resampling serves as the first step to further examine the gravity wave display beyond the apparent impact of the cloud brightness. In Sect. 5 representative cases are chosen to extract a universal law that controls the wave display. As a step further, demonstrations of these cases are shown for verification. Conclusions are given in Sect. 6.

\section{CIPS dataset}

CIPS version 4.20 Level 2 orbital strips of PMC albedo at $83 \mathrm{~km}$ altitude are used in this study (Lumpe et al., 2013). CIPS is one of the two instruments that are currently operating aboard the AIM satellite. The CIPS instrument (McClintock et al., 2009) is a panoramic imager viewing nadir and off-nadir directions to measure ultraviolet radiation (centered at $265 \mathrm{~nm}$ ) scattered by the clouds and atmosphere. In the spectral region near $260 \mathrm{~nm}$, absorption of ozone in the lower atmosphere renders the earth nearly dark, maximizing the contrast of PMC scattering relative to the atmospheric background. It is worth mentioning that, aside from the PMC data used in this study, the CIPS Rayleigh albedo anomaly (RAA) data are also made available to the public. The RAA data characterize the gravity waves at altitudes of $50-55 \mathrm{~km}$ (Randall et al., 2017), serving as the only existing imaging dataset that can reveal the gravity wave horizontal structures near the stratopause. CIPS consists of four wide-angle cameras arranged in a "bowtie" shape that covers a $120^{\circ}$ (along orbit track) $\times 80^{\circ}$ (cross orbit track) field of view (FOV). It measures scattered radiances from PMCs near $83 \mathrm{~km}$ altitude to eventually derive cloud morphology and particle size information. Each individual cloud had a stack of maximum seven exposures from different view angles, from which we derive PMC scattering phase functions and eventually retrieve the nadir horizontal spatial features. CIPS horizontal resolution is approximately $2-7 \mathrm{~km}$ depending on how far a given pixel is from the center of the bowtie, with the center of the bowtie possessing the finest resolution. CIPS version 4.20 retrieval algorithms and data products are described in detail by Lumpe et al. (2013). In Level 1a the camera flat-fielding is applied to remove the pixel-to-pixel variation induced by each camera, and then normalization between the cameras is applied. In Level $1 b$ all cameras are merged to create a consistent set of CIPS measurements. In this stage the measurements are adjusted onto a common grid system of $25 \mathrm{~km}^{2}$ resolution. In the Level 2 processing the cloud scattering signal is further distinguished from the background Rayleigh signal based on their different scattering angle dependence. The Level 2 retrieval is operated on the Level $1 \mathrm{~b}$ data, so that the Level 2 data product is registered on the same $25 \mathrm{~km}^{2}$ resolution grids. Throughout the 10 years of the AIM mission, the CIPS retrieval has experienced earlier versions, and the theoretical framework of these retrievals was described in Bailey et al. (2009). In the previous CIPS data versions the Rayleigh background was retrieved pixel by pixel rather than over the entire orbital strip like in version 4.20. This earlier approach will result in increased retrieval noise in the cloud parameters, requiring additional smoothing procedure to increase the signal-to-noise ratio at the expense of retrieval resolution.

\section{Wave tracking algorithm}

\subsection{Analytical demonstration}

One-dimensional (1-D) continuous wavelet transform (CWT) calculations constitute the basic elements of the proposed CIPS wave tracking algorithm. The term "wave tracking" in the context of this paper refers to the operation of tracking all existing quasi-periodic wave displays in the CIPS PMCs over the scale range of $\sim 20-60 \mathrm{~km}$, rather than tracking a specific wave event. We first demonstrate the effectiveness of the approach using an analytically composed series. When the algorithm is applied to CIPS (see Sect. 3.2 and later), each individual CWT calculation will be carried out along a presumably $\sim 400 \mathrm{~km}$ (or 80 grids) long CIPS PMC albedo segment and will deliver a total of 22 components spanning the scales $2.0-76.0$ in grids ( $5 \mathrm{~km}$ per grid). The relevant scale range for this study is $\sim 4.0-12.0$ grids $(\sim 20-60 \mathrm{~km})$, and the total power of the relevant CWT components is termed "CWT power" or "albedo power" in the following CIPS-related discussion. It is worth pointing out that a $\sim 20-60 \mathrm{~km}$ scale range is focused on in this study because the total albedo power spatial distribution corresponds well with the readily observed wave signatures in the albedo maps (see Figs. 2, 7-9, and 11). The $60 \mathrm{~km}$ threshold appears particular, but it is chosen simply because it is among the sequence of individual scales for the CWT calculations, which are 4.0, 4.7, 5.6, 6.7, 9.5, and 11.3 grid units. A radius of roughly $\sim 400 \mathrm{~km}$ is chosen because it is able to include many repeats $(\sim 10)$ of the wave ridge and trough to provide the full extent of wave display. Yet the spatial span of the wave display should not be overly extended because we do not wish to go across several wave events or different types of variability along the path of CWT calculation. These calculations will be carried out in all $360^{\circ}$ radial directions ( $3^{\circ}$ increment) when being applied to CIPS PMCs. 


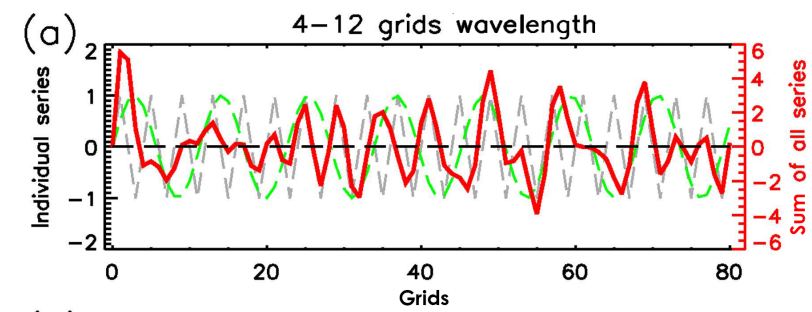

(b) 4-12 grids wavelength
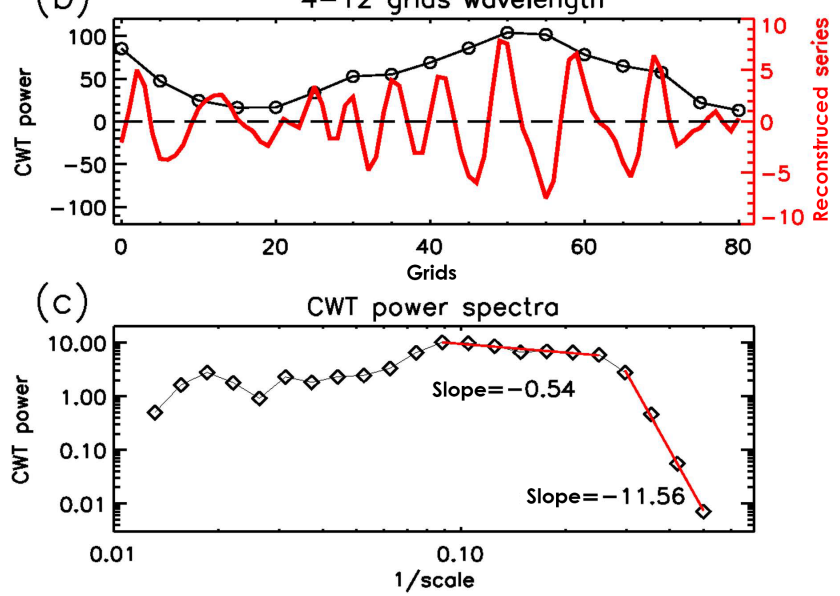

Figure 1. Demonstration of the continuous wavelet transform (CWT) applied to an artificially created series. (a) The created series (red line) consisting of seven sinusoidal components with wavelengths (or scales) of 4.0, 4.7, 5.6, 6.7, 9.5, and 11.3 grid units, each of them with the same amplitude of 1.0. The gray and green dashed lines are for the scales of 4.0 and 11.3 grids, respectively, serving to demonstrate the individual series. Note that the red curve has used the scale on the right axis which corresponds to a 3-times-larger magnitude. (b) The total CWT power series (black with circles) and the reconstructed series (red line) using the wavelet coefficients and components. (c) The CWT power spectra. The slopes (i.e., red lines) are calculated over the seven scales used to create the series and the smaller scales that are present due to the non-orthogonal basis in the CWT calculation.

A 6th-order Morlet wavelet is adopted in this study. A Morlet wavelet (Gabor, 1946) is a complex exponential (plane sinusoidal waves) windowed by Gaussian function so that both periodicity and localization can be realized, defined as $e^{i k x / s} e^{-x^{2} /\left(2 s^{2}\right)}$, where $k$ is the (non-dimensional) order and $s$ is the scale. Scale $s$ determines both the width of the Gaussian function and the period of the sinusoidal signal. In a 6th-order Morlet wavelet $(k=6)$ the scale $s$ is almost precisely the period of the sinusoidal signal. We emphasize here that in the following main analysis applied to the CIPS PMC images the scales of the wave structures refer to the Morlet wavelet scales. However, we will first use an artificially created series to demonstrate that CWT and fast Fourier transform (FFT) deliver qualitatively consistent results.

The artificially created series, which is shown as the thick red curve in Fig. 1a, is composed of seven plane sinusoidal waves that have wavelengths of 4.0 to 12.0 grids and zero ini- tial phases at the start point of the series. These wavelengths are the 5th-11th scales delivered in a CWT calculation mentioned above. The shortest and longest of these plane waves are shown by the gray and green dashed lines in Fig. 1a. The total CWT power over the scales of 4.0-12.0 grids is shown as the black curve in Fig. 1b, whereas the thick red curve in Fig. $1 \mathrm{~b}$ is the reconstructed series using the Morlet wavelets and the corresponding CWT coefficients. It is notable that the black curve is smooth and exactly follows the magnitude change of the localized shorter scale signals. The basis vectors of CWT are not orthogonal, and therefore a reverse CWT does not exist in a strict sense, but we do find that the reconstructed series greatly resemble the original series although their magnitudes slightly differ. This suggests that the CWT and reverse CWT work efficiently on a quasi-periodic signal series. Especially, the fact that CWT almost precisely captures the local variability of the wave amplitude suggests that the CWT algorithm will be an effective approach to detecting the gravity waves in the CIPS PMCs.

Figure 1c shows the CWT spectrum of the created series. We just mentioned that the created series is the sum of only seven FFT components, but since FFT and CWT have different basis vectors, the CWT will project onto all the scales from 2.0 to 76.0 grids. Over the seven relevant scales from 4.0 grids to 12 grids the slope on the double-logarithm diagram is as weak as -0.54 , reflecting the fact that we have adopted identical amplitudes for all the plane sinusoidal waves used to create the series. For scales $<4.0$ grids there is an extremely rapid decrease of the CWT power density with a slope of -12 , indicating that these scales are almost non-existent. It is noteworthy that the created signal exhibits a quasi-periodic nature even though there are no spectral peaks. However, due to the involvement of multiple scales, the fluctuation washes out in a certain portion of the series (i.e., in the range of 3.0-23.0). In this latter case, quasiperiodicity is impaired by the involvement of multiple components.

\subsection{Demonstrations using concentric wave patterns}

In terms of applying the algorithm to the PMC images from CIPS, a direct 2-D CWT routine would be preferred but does not exist in the standard numerical recipe. In addition, there is ambiguity in determining the phases in the CWT algorithm because there is always a tradeoff between the localization of the signal and a clear phase determination.

In this study we used an algorithm based on consideration of expediency as well as efficiency. The 1-D CWT calculations are carried out in all $360^{\circ}$ radial directions $\left(3^{\circ}\right.$ increment) centered at a given location within the CIPS albedo orbital strip. The resampling of the CIPS data is performed in the radial and angular directions centered at such a location. In the radial direction the increment step is $5 \mathrm{~km}$, which is the same as the CIPS Level 2 resolution, while in the angular direction a step of $3^{\circ}$ is chosen based on the consideration 
of yielding a sufficiently detailed yet smooth spatial map of the albedo wavelet power. This approach was inspired by the intent of detecting ideal concentric waves because such a design will result in the maximum CWT albedo power within a given radius. In addition, performing the CWT in all radial directions will efficiently capture the waves of all orientations. Since the basis vectors of 2-D FFT are straight (linear) waves of all orientations, the current algorithm is more or less a short version of the 2-D FFT in a localized area but has the merit of being straightforward in reflecting the local albedo wave power.

The specifics of the algorithm are described as follows. An elliptical region of 80 grids along-track and $80 \times 0.65$ grids cross-track is used to carry out the CWT calculations. The factor 0.65 is empirically chosen based on a few fitted examples of the concentric waves found in CIPS. The rationale of such a factor will be revisited in a following paragraph. Concentric waves found in CIPS appeared to be mostly elongated in the along-track direction. This factor has no qualitative effect on the results except that, when the elliptical region fits an actually existing concentric wave pattern, it achieves the largest albedo CWT power, which is a desired condition for the wave tracking operation. The elliptical region is moved around to fully cover the orbital strips. The steps of the movement are the half axial lengths in both along-orbit and crosstrack directions. Except for the intent to achieve full coverage of the orbital strip, the "move-around" scheme will also ensure the capture of the albedo CWT power from varying orientations in the same region. The albedo CWT power map enclosed in the given elliptical region measures the total albedo fluctuation intensity in the scale range of $\sim 20-60 \mathrm{~km}$ for each spatial location.

Five examples of concentric waves and the corresponding albedo power maps are presented in Fig. 2 to demonstrate how the wave tracking algorithm works for CIPS. Concentric waves are chosen because they are well documented as possessing a unique morphology and meanwhile serving as a proof of the connection between the lower and higher atmosphere. These waves are extremely rare and were detected only a few times for a given PMC season. The percentage of detection is less than $5 \%$ in terms of days per season, and by spatial coverage the fraction is even smaller. All examples have shown only partial rings. The model results by Vadas et al. (2009) simulating the concentric rings to compare with the observations near Fort Collins, Colorado, have shown that including realistic zonal winds can substantially disrupt the completeness of rings in the mesosphere, with about $50 \%$ of the wave structure being disrupted. In the CIPS PMCs the rings are more severely disrupted. In some cases only a $20^{\circ}$ section of the full $360^{\circ}$ circle has survived (not shown). The model results by Vadas et al. (2009) also indicate that if a July zonal wind is adopted in the simulation the rings will be elongated to an axial ratio of about $0.6-0.7$, which roughly agrees with the findings in CIPS. It appears that in summer the concentric waves will likely be substantially elongated compared to those in winter or spring.

The example shown in Fig. 2a has a wavelength of about $60-80 \mathrm{~km}$, which is longer than most other concentric waves in the CIPS PMCs, but since the (bright) ridges are much narrower than the (dim) troughs the albedo power still reaches notable magnitudes. Remember that in this study only the CWT power within the wavelength range of $\sim 20-60 \mathrm{~km}$ is calculated, and what is shown in Fig. 2a again reminds us that wave patterns in PMCs only achieve quasi-periodicity. The example in Fig. $2 b$ is a set of highly distinct concentric waves. The albedo power reaches notably larger values when the waves are the most distinct in the upper-right quadrant of the albedo map (see Fig. 2b, upper panel). In the upperleft quadrant of the albedo map the waves become blurry, but one can still tell that they are concentric. In the blurry part of the map the albedo power decreases sharply, as is clearly seen from the corresponding lower panel. Given the confined scale range of $\sim 20-60 \mathrm{~km}$, being visually blurry can be interpreted as possessing a lower variance level, which may have been a result of diffusive processes. In the spectral space, if the leading scales stand out poorly from the neighboring smaller scales, then the structure will be less organized, which also makes it less distinct. This topic will be further addressed in Sect. 5. Within the same wave display, varying from being distinct to blurry must be controlled by some physical process yet to be unraveled. In the lower-right quadrant of the albedo map there are some straight waves that have cross-interfered with each other but are much less distinct than the main part of the concentric waves. This suggests that multiple wave packets of different morphology often coexist right next to each other in the PMCs. Figure 2c shows an example of extremely faint concentric waves which are characterized by much lower albedo power than those in the rest of the examples. Figure $2 \mathrm{~d}$ shows a slightly weaker but also fairly distinct partial concentric wave pattern that also coexists with some blurry straight-wave patterns. Figure $2 \mathrm{e}$ is an example of concentric waves in a brighter background that occurred in 2009. In 2009 CIPS orbits appear twisted due to the camera's turned position.

Lastly, we must also point out an inherent drawback of the CWT wave tracking algorithm. It is apparent that in all the examples shown in Fig. 2 the clouds (e.g., $>5.0 \times 10^{-6} \mathrm{sr}^{-1}$ ) do not fill up the entire elliptical region, and furthermore the wave signatures are mostly partial rings. If such an inhomogeneity is strong, the mean albedo power or background brightness can misrepresent the characteristics of the region. We therefore have chosen a relatively small radius $(\sim 400 \mathrm{~km})$ to carry out the calculations to minimize the effect of inhomogeneity. Due to the high complexity of the PMC signatures, it is unlikely to simply eliminate such an effect. Nevertheless, so far we have not run into any noteworthy problem due to such effect when analyzing the wave tracking results. 

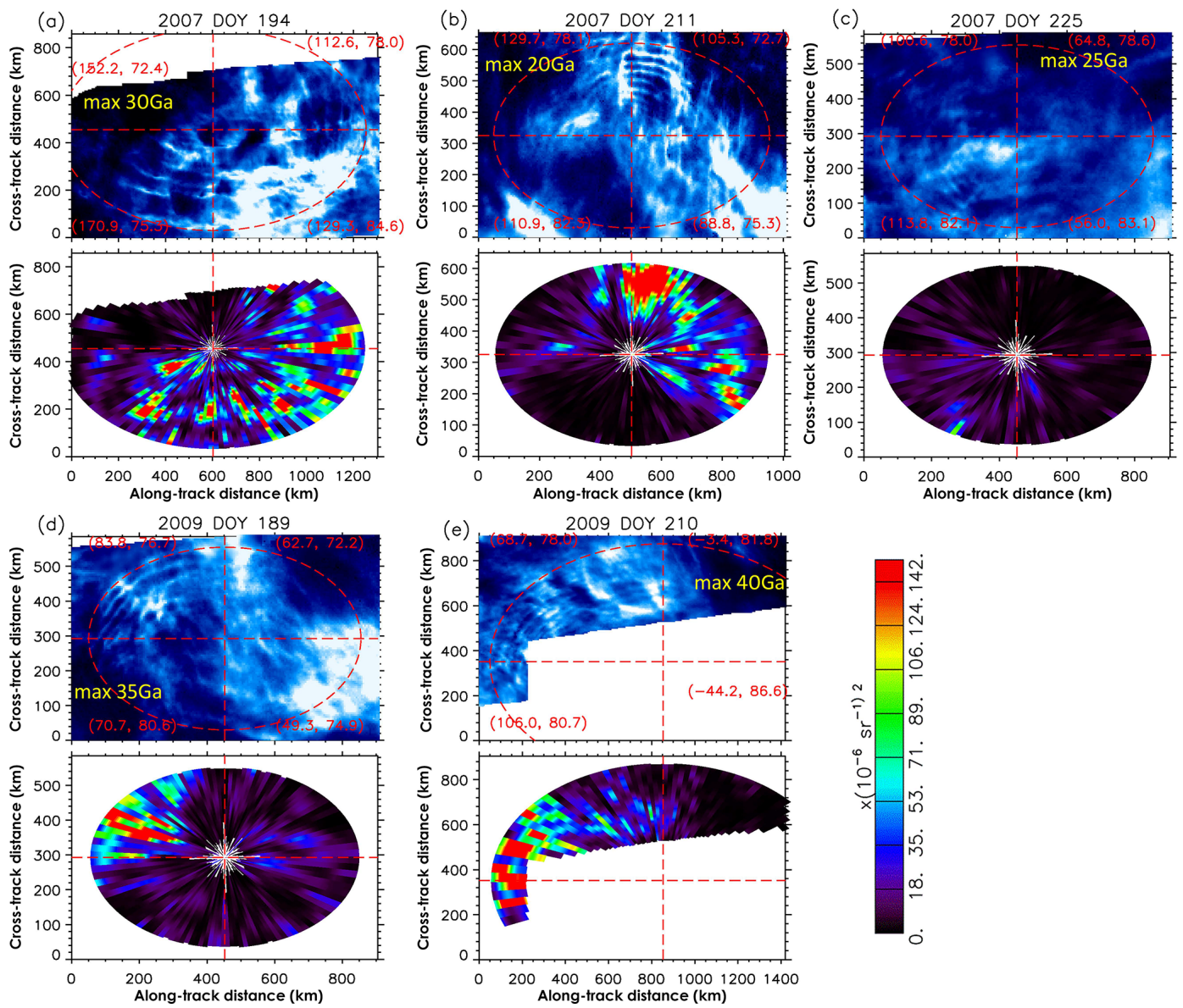

Figure 2. Wave tracking algorithm applied to the concentric waves (a)-(e) in the CIPS orbital strips. Wave tracking is carried out within the elliptical regions. For each pair, the upper panel is the albedo, and the lower panel is the albedo CWT wave power field by $3^{\circ}$ angular bin $\times 5 \mathrm{~km}$ radial bin. Blue-white color scheme is used for the albedo maps, with the white color representing the maximum albedo values indicated by the yellow legends, with $1 \mathrm{Ga}=1.0 \times 10^{-6} \mathrm{sr}^{-1}$. The red numbers at the four corners are longitudes and latitudes. The rainbow color bar for the CWT power is used universally in this paper.

\section{Statistics of the gravity wave albedo power values}

\subsection{Brighter PMC background threshold}

The statistical ensemble consists of the albedo power values averaged within all elliptical regions used to carry out the wave tracking. We need to emphasize here that the albedo power refers to the total power within the scale range of $\sim 20-60 \mathrm{~km}$. The wave tracking procedure has been carried out throughout the two northern hemispheric summers in 2007 and 2010 from 1 June to 31 August. We take a particular interest in the wave display in the brighter PMCs because the previously identified waves mostly reside in the relatively dim cloud environment. We split the cloud population into two subsets, one containing only $0-2 \%$ bright clouds (with a threshold of $25 \times 10^{-6} \mathrm{sr}^{-1}$ ) in the elliptical region, which is the overall dimmer cloud group, and the remaining set containing a systematically larger fraction of bright clouds. The $25 \times 10^{-6} \mathrm{sr}^{-1}$ threshold is chosen empirically. The bright- cloud presence frequency (denoted by freq ${ }_{25}$ hereinafter) is a better index than the mean cloud albedo in characterizing a systematically brighter cloud background.

\subsection{Histograms of the albedo power values}

Figure 3 shows the histograms of the albedo power values for the full set of wave detections (in red) and those residing in the brighter background (in black), and both show a peak number density being close to zero and then a rapid decrease as the albedo power increases. Peak locations approaching zero indicate that the majority of the waves are in the range of low albedo power. It is especially worth noting that the full set and the brighter set collapse as the albedo power is greater than $\sim 20 \times 10^{-12} \mathrm{sr}^{-2}$, whereas before this point they separate substantially. This indicates that brighter clouds mostly coincide with the higher albedo power and that the waves that have caused the difference in the two curves reside in the dimmer group. In addition, the collapsing part of the curves 


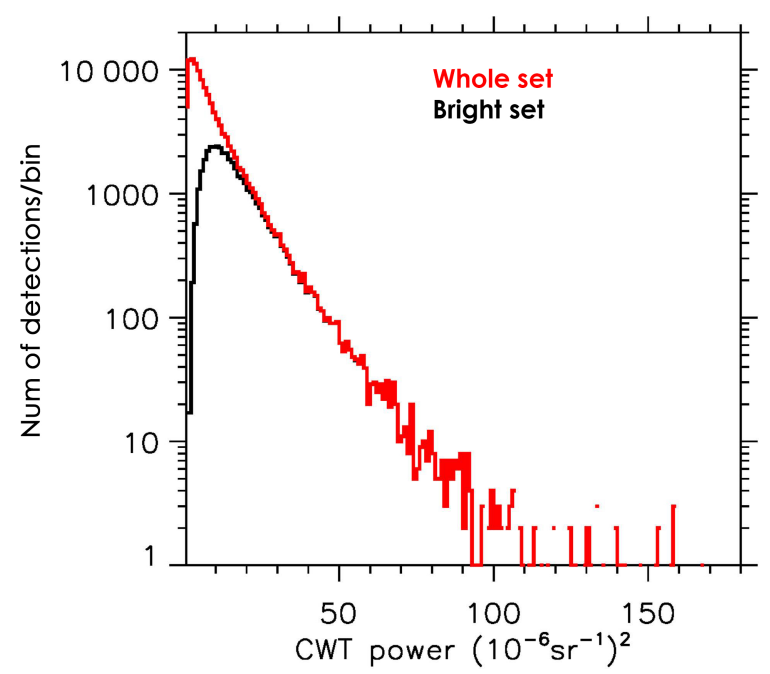

Figure 3. The histograms of the CWT power values obtained throughout the two northern hemispheric summers in 2007 and 2010. Each CWT power value refers to an average within a given elliptical region. The black curve is for the brighter cloud group that corresponds to freq $25>2 \%$ within a given elliptical region, and the red curve is for the full set of detections. The bin size within which we count the detection number is $1.0 \times 10^{-12} \mathrm{sr}^{-2}$.

forms a straight line under a logarithmic vertical axis, suggesting an exponential decrease of the wave detection number density as the albedo power exceeds the value where the peak number density occurs. For the full set of the wave detections the straight line proceeds to a much smaller albedo power value, while for the brighter set it shows a peak at a higher albedo power. Removal of the dimmer cloud group by a given threshold caused this. In general, the exponential decrease of the wave detection number density toward increasingly higher albedo power is a robust result, but how rapidly the number density decreases may show interannual variability (not shown). It is worth mentioning that the analysis of the PMC ice water content measured by the Solar Backscatter Ultraviolet (SBUV) instruments (DeLand and Thomas, 2015) yielded the same type of distribution. These authors further investigated the apparent interannual variability of the distribution slope and concluded that population ratios between the hierarchy of particle sizes may have been different for individual years to cause this variability. Later in this paper we will find that PMC albedo and the corresponding wave power hold a statistically linear relationship, and therefore it is within expectation that both the PMC intensity and the wave power follow similar distributions. Figure 3 also shows that when the albedo power reaches its upper limit the curve flattens out, but such a behavior is simply caused by the low sample number, which is of no significance.

\subsection{Relationship between the albedo power and freq 25}

Although the dimmer subset takes up a major fraction of the cloud population, which exceeds $65 \%$, we take just as much interest in the wave display in the brighter cloud background in this study. Figure 4 shows the scatterplot of the wave detections in the brighter cloud background on the plane of albedo power versus freq25. In Fig. $4 a$, within equally spaced bins of freq $_{25}$ and the albedo power, the rainbow-colored squares represent the number density of the wave detections. The dimmer group of PMCs are collapsed into the first bin of freq $_{25}=0-0.02$ and is not shown in this scatterplot to avoid any discontinuity induced by the artificially chosen threshold (i.e., $25 \times 10^{-6} \mathrm{sr}^{-1}$ ).

Figure 4a shows that the wave detections (see rainbow colors) are grouped more densely in the low-albedo-power as well as the low-brightness region. This generally agrees with what Fig. 3 shows, but we should note that the wording "low" is in a relative sense because we have taken away the dimmer set in this analysis. For any given $\mathrm{freq}_{25}$ the wave detection number density distribution resembles a normal distribution, but the outliers are strongly asymmetric, showing a muchfurther-reaching albedo power value at the upper limits. In addition, we find that as freq $_{25}$ increases the peak number density moves toward an increasingly larger albedo power, shown by the dashed black curve. Both suggest that in a statistical sense larger albedo power corresponds to brighter cloud background. At the dimmer end where freq $_{25}=0.02$ the albedo power is within $50 \times 10^{-12} \mathrm{sr}^{-2}$ (amplitude $7.0 \times 10^{-6} \mathrm{sr}^{-1}$ ), while for freq $_{25}=1.0$ it reaches values greater than $150 \times 10^{-12} \mathrm{sr}^{-2}$ (corresponding to an amplitude of $\sim 12.0 \times 10^{-6} \mathrm{sr}^{-1}$ ).

We next adopt an analytic form to parameterize the relationship between the albedo power and freq $_{25}$ in order to resample the wave detections into a consistent normal distribution. This is a preliminary step taken for the future removal of the apparent dependence of the wave power on the background cloud brightness. The mechanism that controls such an apparent dependence is not pursued in this paper, and a future study will be required to understand this since we have learned that the previous modeling studies do not seem to directly interpret it. For example, Chandran et al. (2012) have shown that both the short-period and longperiod gravity waves ultimately reduce the domain-averaged PMC brightness.

A set of square root sectioning curves (albedo power $=$ factor $\times \sqrt{ }$ freq $_{25}$ ) are used to split the wave detections into subsets to achieve the normal distribution. Such an analytic form is chosen because it roughly coincides with the dashed black curve in Fig. 4a that shows how peak number density of wave detections varies with freq 25 . The interval of the sectioning curves is by a factor of $1 / 2^{i / 4}$, with index $i$ varying from 0 to 21, as shown in Fig. 4a. In total, 23 sections are used to produce a smooth probability distribution, with the first index $i=-1$ being the closest to the 

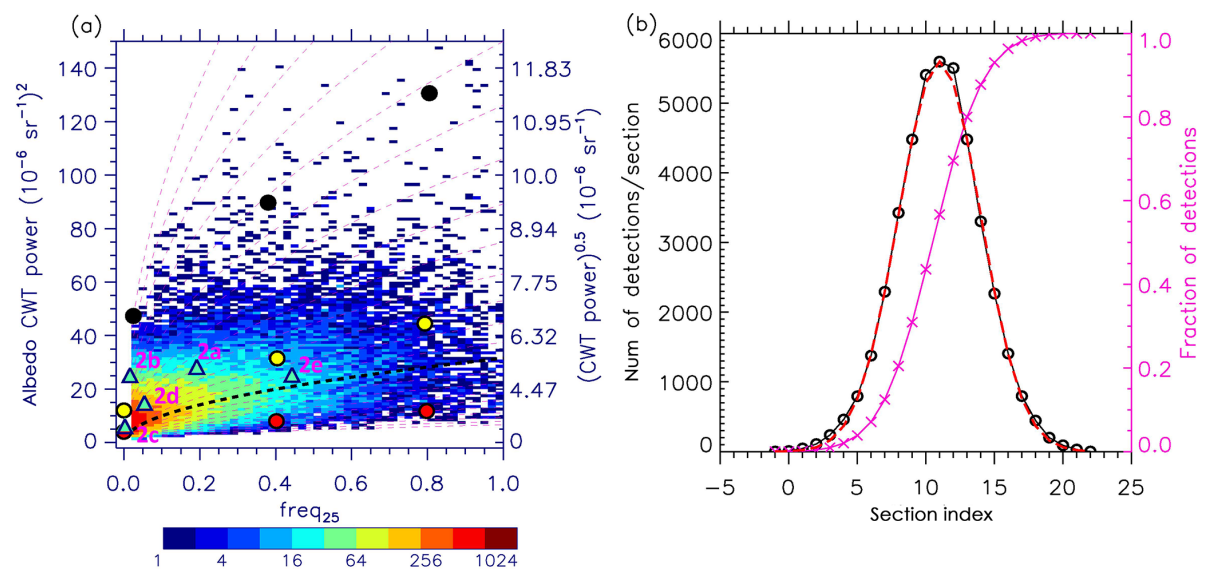

Figure 4. (a) Scatterplot of the wave detections to reflect the relationship between the bright-cloud frequency (freq 25 ) and the albedo CWT power. Only detections corresponding to the brighter clouds (freq $25>0.02$ ) are included. The rainbow colors are the detection number density within each bin $\left(\Delta\right.$ freq $25=0.02$, and $\left.\Delta \mathrm{CWT}=1.0 \times 10^{-12} \mathrm{sr}^{-2}\right)$. The dashed thick black lines roughly follow the peaks of the detection number density isopleths, serving to derive an analytic relationship between the cloud brightness and the CWT power. The turquoise triangles correspond to the concentric waves shown in Fig. 2. The colored dots are selected roughly at the two tails and the peak of the normal distribution and at three brightness levels of freq $25=0,0.4$, and 0.8 . These nine selections are representative of the full set of wave detections, to further demonstrate the wave exhibition in Figs. 7-9. Using a sequence of analytic curves (magenta dashed) to resample the wave detections, we obtain a normal distribution as is shown in (b). In (b) the black curve with the circles represents the obtained distribution from the left panel, while the dashed red curve is the Gaussian fit. The magenta curve with crosses is the accumulated fraction of the data points.

albedo power axis. The $2^{i / 4}$ rather than a linear sequencing is chosen to account for the fact that the data points become increasingly denser from being close to the albedo power axis toward the freq 25 axis. Figure $4 \mathrm{~b}$ confirms that the resampling produces almost a precise normal distribution, and at the 11th interval it reaches the peak, which splits the wave detections in half.

\subsection{Relationship between the albedo power and mean albedo}

In this subsection we reexamine the albedo power dependence on the background cloud brightness using the mean albedo within the elliptical region as the horizontal axis. This angle of investigation provides a smoother picture because it does not use any imposed threshold $\left(25 \times 10^{-6} \mathrm{sr}^{-1}\right)$. Two purposes are served by doing so. First, we tend to include the wave detections with dimmer background since they take a major fraction of the cloud population as is mentioned above. Second, we tend to examine whether the full set of wave detections and those residing in the brighter cloud background follow a consistent statistical relationship between the albedo power and the background cloud brightness.

Figure 5a shows a similar scatterplot except using the mean albedo (within the elliptical region) as the horizontal axis. The isopleths of the wave detection number density suggest a linear relationship between the albedo (fluctuation) power and the background albedo, and we also note a strong asymmetry of the albedo power distribution between the lower and upper limits, suggesting an apparent dependence of the albedo power on the background mean albedo. This confirms that albedo power monotonically increases with the background cloud brightness, except that a different analytic form will be used to carry out the sectioning procedure.

Linear sectioning lines are applied to the plane of the albedo power versus the mean background albedo to yield a normal distribution. The sectioning lines are emitted from the $(0,0)$ point, and the angular interval gradually increases from the lower-right to upper-left corner to achieve symmetry of the distribution. A total of 27 intervals are used. Such a sectioning and resample procedure makes both the full set and the dimmer subset achieve the normal distribution, shown in Fig. $5 \mathrm{~b}$, which confirms a consistent behavior between the dimmer set and the full set. The peak of the normal distribution is reached at the 12th sectioning interval. Both Fig. 5 and Fig. 3 (i.e., the albedo power histograms) indicate that the dimmer subset and the full set follow a similar behavior.

\subsection{Representative cases on the scatterplot}

The dots of three different colors in Fig. 4a are the sample selections of the wave detections for future-demonstration purposes. Wave tracking yields a large number of detections, and we aim at obtaining a universal law that governs the full set of wave display. The selections are made roughly at the two tails and the peak of the normal distribution (Fig. 4b), labeled the high-, medium-, and low-albedo-power categories. Note that these categories are chosen based on the combina- 

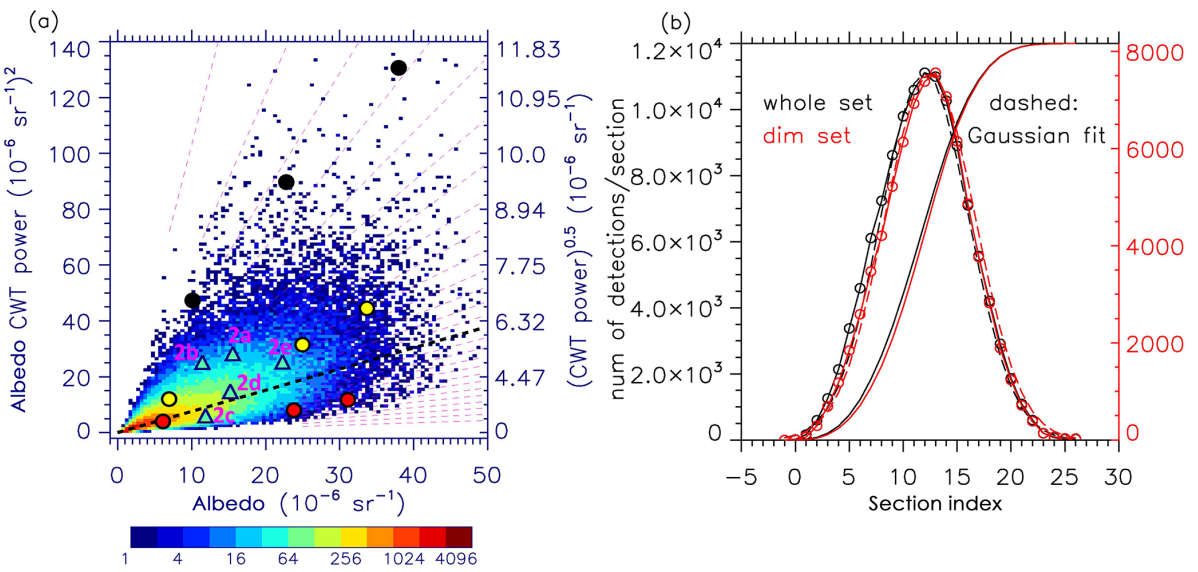

Figure 5. (a) Same as Fig. 4 except for using both dimmer and brighter sets of detections and use the mean albedo within the elliptical region as the horizontal axis. The bin of albedo is $0.5 \times 10^{-6} \mathrm{sr}^{-1}$. The turquoise triangles and colored dots are the same as in Fig. 4. (b) The normal distributions for both the dimmer subset and the full set of wave detections. The curves without symbols are accumulated fraction of detections with a vertical axial range of $0-1.0$ (not shown), which is the same as in Fig. $4 \mathrm{~b}$.

tion of both albedo power and the background cloud brightness. Three brightness levels (freq $25=0,0.4$, and 0.8 ) are used, and therefore a total of nine selections are made. The cases at freq ${ }_{25}=0.4$ and 0.8 almost precisely follow the sectioning curves. While at freq $_{25}=0$, the three selections are made based on a linear relationship with the two selections at freq $_{25}=0.4$ and 0.8 . This is because the dimmer subset (freq $25<0.02$ ) is not included in Fig. 4a. We should point out that the sectioning curves serve only as the guidance to select the cases that reasonably cover the full set of detections, but our conclusions are not sensitive to what exact cases are chosen.

We place the same nine selected cases in Fig. 5a and find that the three albedo power categories also roughly follow the linear sectioning lines and are also located approximately at the lower and higher tails and at the peak of the normal distribution.

At last we check on where the concentric waves shown in Fig. 2 are distributed on the scatterplots. From looking at the turquoise-colored triangles we find that they do not seem to preferably occur at any specific combinations of albedo power and background brightness. Rather, the five cases are approximately evenly spread over the core region, and none has occurred in either high-albedo-power or high-background-brightness ranges. Also, it is worth mentioning that the case of the most distinct concentric waves shown in Fig. $2 b$ has a combination of freq $25=0.016$ and albedo power $=25.4 \times 10^{-12} \mathrm{sr}^{-2}$, suggesting that the combination of relatively low background brightness and relatively high albedo power seem to correspond to the best clarity of wave display. Although rare in occurrence and possessing a known particular form of driving mechanism, concentric waves seem to have shown a regular behavior in terms of the correspondence between the albedo power and the background cloud brightness.

\section{Wave displays of the representative cases}

\subsection{Albedo power spectra}

The albedo power spectra of the nine selected detections are shown in Fig. 6, with the three brightness levels being collapsed to each other for each albedo power category. These power spectra are calculated to extract a universal law that governs the wave display. The power spectra of all orientations (full $360^{\circ}$ with $3^{\circ}$ step) within a given elliptical region are averaged for each individual scale in the range of $\sim 20$ $60 \mathrm{~km}$. Although inhomogeneity exists between different orientations, the spectra of the main wave signatures will dominate the mean spectrum. Unlike the FFT power spectra that are prone to exhibiting spikes (not shown), the CWT power spectra have blunter features. Especially, due to the spatial average it is even less likely that spectral peaks will stand out unless a given scale within the $\sim 20-60 \mathrm{~km}$ range is consistently dominant throughout the gravity wave population, which is not the case.

Based on the nine representative cases, the general form of power spectra can be expressed as $A \times(1 / \text { wavelength })^{\alpha}$, where coefficient $A=1.42 \times 10^{-4}, 1.45 \times 10^{-4}$, and $1.49 \times$ $10^{-4}$, and the spectral exponent $\alpha=-2.5,-2.9$, and -3.2 for the low-, medium-, and high-power categories, respectively. Note that the three categories are defined in Sect. 4.5. For a confined range of wavelengths, i.e., $\sim 20-60 \mathrm{~km}$, the higher wave power or larger variance level of the display will correspond to higher clarity of wave display or to sharper features. More rapid decay toward the smaller scales will also contribute to higher display clarity because the leading scale will be more dominant over the smaller scales, and therefore the wave signature will be better organized. The actual wave displays shown in Sects. 5.2 and 5.3 will verify these arguments. 

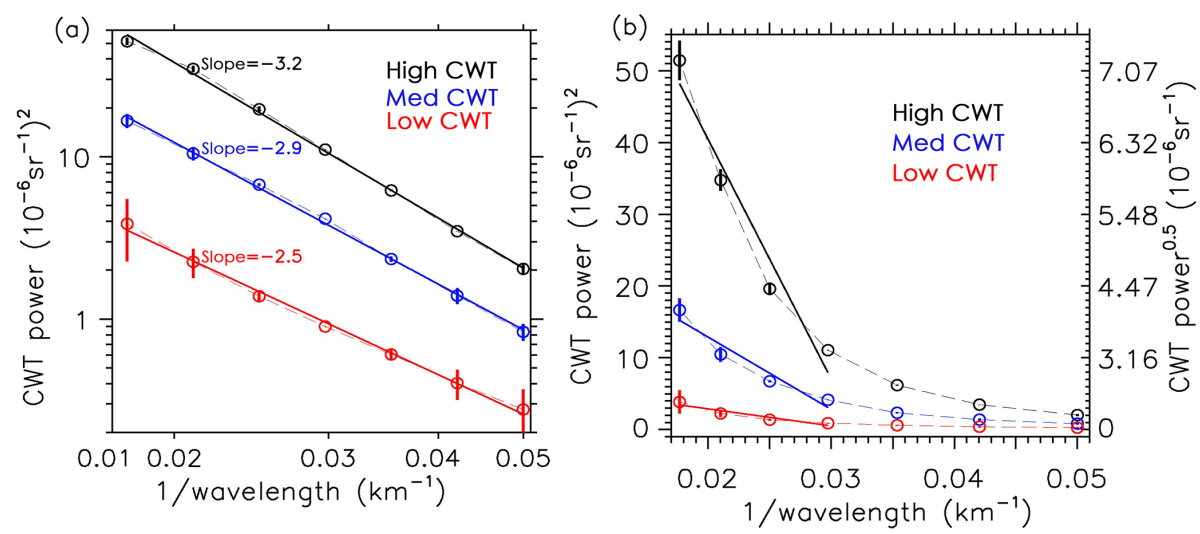

Figure 6. Albedo CWT power spectra for the nine detections selected in Fig. 4. The black, blue, and red colors correspond to the uppermost row (high albedo power), middle row (medium albedo power), and the lowermost row (low albedo power) of dots in Fig. $4 \mathrm{a}$, respectively. For each albedo power category the CWT power spectra are normalized to collapse within the error bars as the $1 \sigma$ standard deviation. The exact match occurs at the medium scale ( $\sim 33 \mathrm{~km}$ wavelength or 0.03 spatial frequency) to obtain the mean slope. The thin dashed lines are original curves, and the thicker solid lines are linear fitting lines. (a) Exponents of decay are $-3.2,-2.9$, and -2.5 for the three categories. (b) Under linear axes it is shown that higher albedo power and larger exponent correspond to more rapid decay of the albedo power toward smaller scales, reflected by the linear fitting lines for the first few dominant scales.

The black lines in Fig. 6 are for the high-power category (see the black dots in Fig. 4a). The three brightness levels possess a consistent exponent $\alpha=-3.2$, and therefore via simple adjustment of $A$ (in the analytic form) a normalization or collapse between the three spectra is achieved. The factors of normalization toward the brightest level are defined as follows:

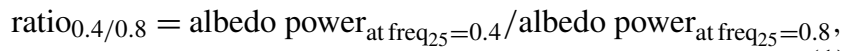

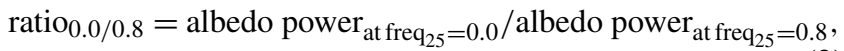

where albedo power refers to the total wave power over the scales of $\sim 20-60 \mathrm{~km}$. As is argued above, the normalization is carried out to examine the wave morphology beyond the wave power dependence on the background brightness. After applying these factors, the three brightness levels will achieve a nearly fully collapsed power spectrum and exhibit the same level of display clarity (see Sect. 5.2). The black line under the linear axes shown in Fig. $6 \mathrm{~b}$ indicates that the high-power category possesses both the highest overall power level and the most rapid decay rate toward the smaller scales.

The error bars in Fig. 6 are the uncertainty ranges over the three brightness levels for the different scales, and the exact match occurs at the middle data point (i.e., $\sim 33 \mathrm{~km}$ of wavelength or 0.03 of spatial frequency). It is noteworthy that both the high- and medium-albedo-power categories have very small error bars, suggesting a strong collapse of spectra at the three brightness levels. The low-albedo-power category, however, has notably larger error bars. This means that when the albedo power reaches very low values the exponent $\alpha$ maintains poorer consistency between the three brightness levels. Measurement noise may play a role in causing this. For waves of small amplitudes and especially with very dim background, the noise will be strong enough to affect the determination of the wave amplitude.

\subsection{Wave displays for the different albedo power categories}

Before examining the wave displays, we first set the rules of presentation. First, a white-blue color scheme with a linear red-green-blue (RGB) code system is used to generate the color bars. Second, the mean albedo within the elliptical region, or the background cloud brightness, is subtracted. Third, the maximum and minimum albedo deviation is set to be $\pm 20.0 \times 10^{-6} \mathrm{sr}^{-1}$ for freq $25=0.8$ and is then reduced by factors $\sqrt{ }$ ratio $_{0.4 / 0.8}$ and $\sqrt{ }$ ratio $_{0.0 / 0.8}$ for freq $25=0.4$ and freq $_{25}=0.0$, respectively. As is argued above, these factors are expected to unify the display clarity between different brightness levels.

\subsubsection{The high-albedo-power category}

Figure 7 presents the CIPS albedo maps and the albedo power maps within the elliptical region for the high-albedopower category (corresponding to the black dots in Fig. 4a). We observe generally widespread and distinctly clear semiorganized structures at all three brightness levels. The three panels show very similar levels of display clarity because their albedo power spectra are almost fully collapsed with the factors $\sqrt{ }$ ratio $_{0.4 / 0.8}$ and $\sqrt{ }$ ratio $_{0.0 / 0.8}$ being applied. The high-power category obviously possesses the overall highest power level, and furthermore it also has the most rapid albedo power decay rate (Fig. 6b) toward the smaller scales, i.e., with $A=1.42 \times 10^{-4}$ and $\alpha=-3.2$, respectively. Both 

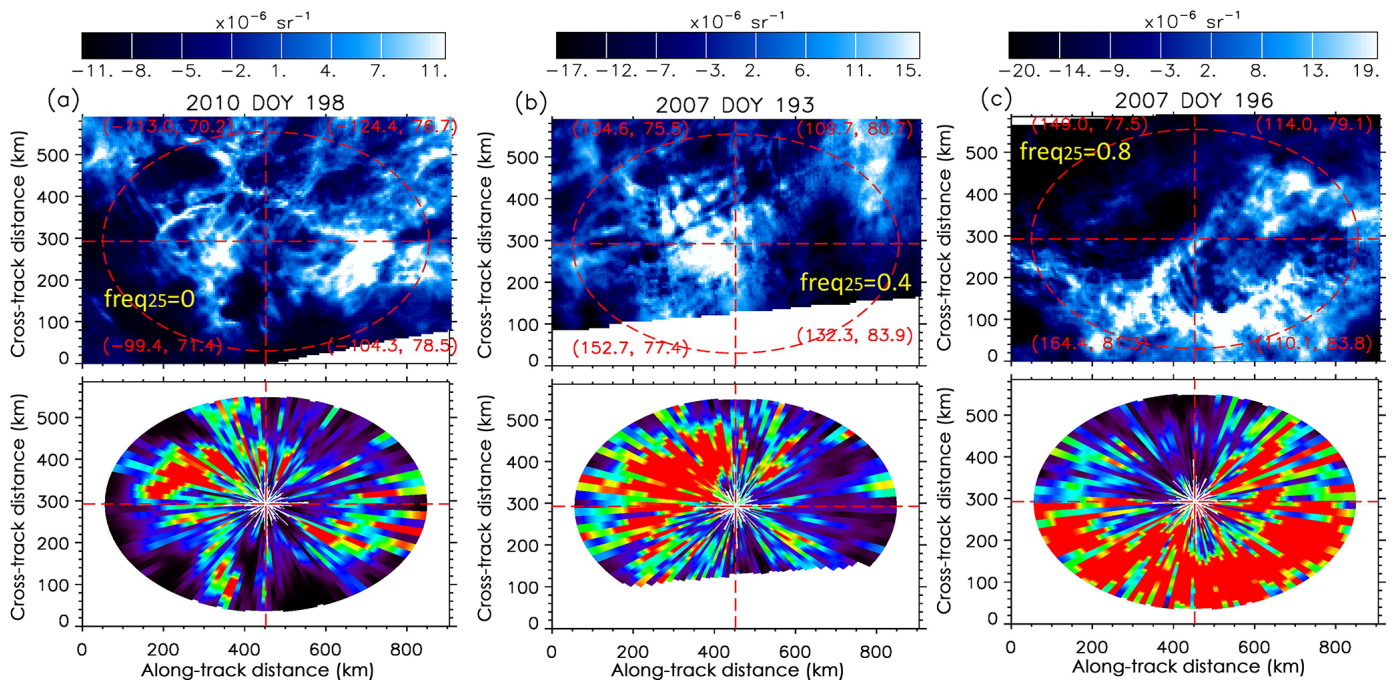

Figure 7. Albedo maps and the albedo power maps corresponding to the high-power category at three different brightness levels, each with the mean background albedo subtracted. The blue-white color scheme used linear RGB color code distribution. The albedo power maps used the same rainbow color bar as in Fig. 2. Based on the factors used to make the albedo power spectra collapse between the different brightness levels, which are ratio $0.4 / 0.8=1.44$ and ratio $0.0 / 0.8=3.13$, in this case (see Eqs. 1 and 2 in Sect. 5.1), the corresponding color bar maxima are reduced by factors $\sqrt{ } 1.44=1.2$ and $\sqrt{ } 3.13=1.77$, to achieve the same level of display clarity.

conditions contribute to the fact that these displays are the most distinct among the three categories. In terms of morphology, the wave signatures resembled straight waves or interference of the straight waves, but occasionally the straightwave features show curvatures at certain portion of the display (in Fig. 7b and c). Overall, the wave morphology is qualitatively consistent regardless of the brightness levels. However, looking more closely, we do perceive a minor difference between the low- and high-brightness display. That is, at the dimmest level (freq $25=0.0)$ the wave display appears to exhibit stronger linearity than those in the brighter backgrounds. From Fig. 2b we did notice that a dimmer background has supported highly distinct wave structures that resembled linear waves. On the contrary, the displays at freq $_{25}=0.4$ and freq $25=0.8$ seem mildly turbulent-like.

\subsubsection{The medium-albedo-power category}

Figure 8 presents the maps for the medium-power category (corresponding to the yellow dots in Fig. 4a). Remember that these cases are the closest to the peak of the normal distribution and therefore are the most representative of the full set of wave detections. The maps shown in Fig. 8 are significantly blurrier than those in Fig. 7, but the wave signatures remain well organized. In the low-brightness end freq $_{25}=$ $0.0)$ there are interfering straight waves approximately oriented perpendicularly to each other. At freq $25=0.4$ the features resemble one-directional straight wave signatures. In the high-brightness end $\left(\right.$ freq $_{25}=0.8$ ) there are knot-like signatures which are apparently deviated from the typical linear wave signatures. Again it is worth mentioning that the three brightness levels are considered to have the same level of display clarity due to the collapse of their CWT power spectra.

\subsubsection{The low-albedo-power category}

Wave displays in the low-albedo-power category (see Fig. 9) provide firm proof that the display clarity of the wave signatures becomes increasingly poorer as the albedo power decreases. All panels show highly blurry features, and yet the orientations of the wave signatures remain recognizable. Note that in this category normalizing the clarity level between different brightness levels has run into larger uncertainty because of the larger error bars (see Fig. 6a). It is noticeable that the wave display for freq $_{25}=0$ seems the most blurry because under this condition both the background mean albedo and the albedo disturbances are likely strongly affected by the measurement noise. Although display clarity does not hold any absolute physical meaning, we can conclude that the gravity wave signatures of $\sim 20-60 \mathrm{~km}$ wavelength become increasingly more diffusive as the corresponding albedo power decreases. Note that Fig. $2 \mathrm{~b}$ shows that even within the same elliptical region $(\sim 400 \mathrm{~km}$ range $)$ the wave display clarity differs drastically. This could be due to the unknown local forcing mechanisms that have exerted different levels of diffusion on the PMC albedo structures. So far we have come to an understanding that semi-organized structures seem extremely widespread, with a hierarchy of different albedo power levels. This is against the belief that a wave detection procedure should yield unequivocal results. 

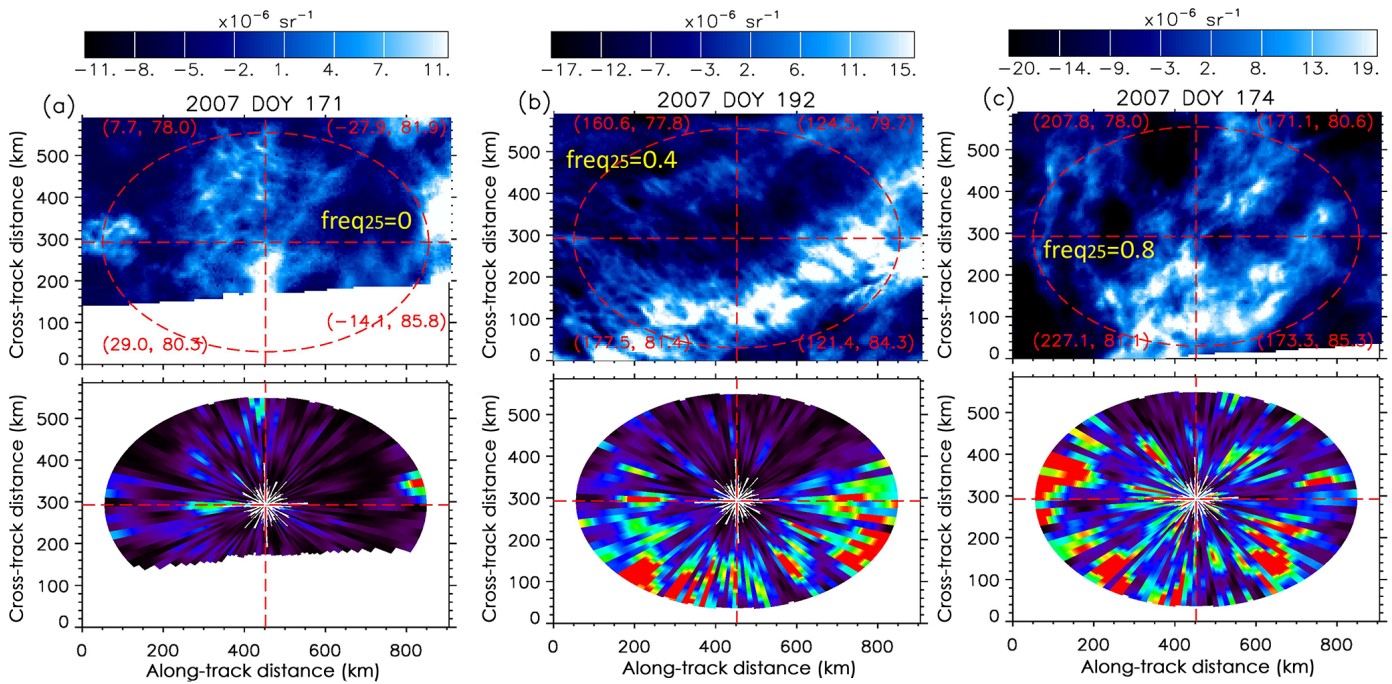

Figure 8. Same as Fig. 7 except for corresponding to the medium-albedo-power category. They are systematically blurrier than those in Fig. 7.
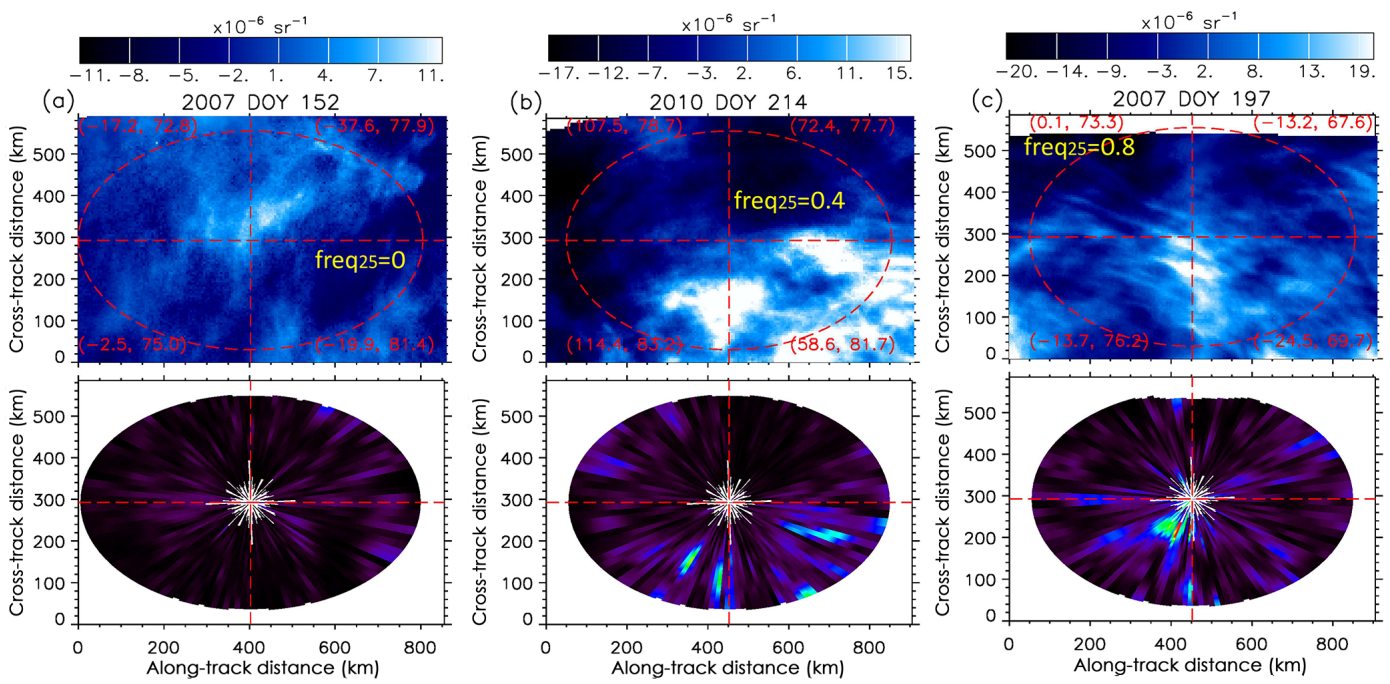

Figure 9. Same as Fig. 7 except for corresponding to the low-albedo-power category. They are furthermore blurrier than what is shown in Fig. 8.

\subsection{Artificially raising the medium toward high albedo power}

The three lines with different colors in Fig. 6a have a hierarchy of albedo power levels as well as different slopes characterized by varying $A$ and $\alpha$. They, however, appear parallel to each other because the standard deviation of the exponent $\alpha(-3.2$ to -2.5$)$ only reaches 0.3 . We next systematically raise the medium power level to match the high power level by increasing the coefficient $A$ but maintain the exponent $\alpha$ to examine how the display clarity improves. We have argued that parameters $A$ and $\alpha$ both have a control over the display clarity. Carrying out this experiment is a way to test the role of the coefficient $A$ in determining the display clar- ity. The previously determined $A$ for the medium-power category is $1.45 \times 10^{-4}$, and in this experiment a new parameter $A_{\text {new }}=2.778 \times A$ is used to draw closer the spectra of the medium toward the high power levels. The factor 2.778 is chosen to maximally match the high- and medium-power spectra over the scales of $\sim 20-60 \mathrm{~km}$.

The result of the amplified case is shown in Fig. 10, and we used the case presented in Fig. 8a to conduct this experiment. Figure 10a shows the albedo map and the power map for the amplified case, and Fig. 10b and c are repeats of Figs. $8 \mathrm{a}$ and $7 \mathrm{a}$, to make comparisons. It is shown that Fig. 10a exhibits notably improved clarity compared to its previous version, and as a result Figs. 10a and c show clar- 

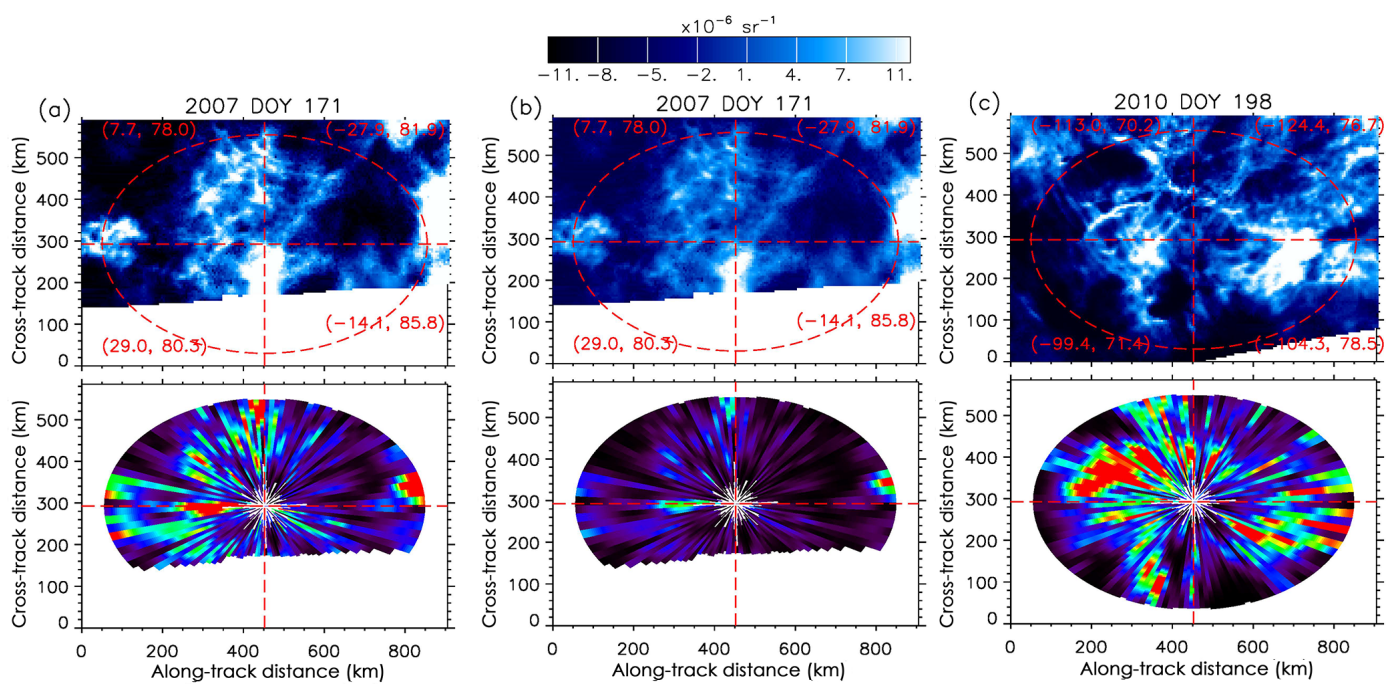

Figure 10. (a) demonstrates that amplifying the waves shown in Fig. 8a (current panel b) by a constant factor $\sqrt{ } 2.778$ (see text in Sect. 5.3) enhances its display clarity significantly. The enhanced display clarity is drawn closer toward what Fig. 7a (current panel c) shows. Note that Fig. 7a belongs to the high-albedo-power category and has the highest level of display clarity.

ity levels drawn closer, but the amplified case is still blurrier. This indicates that the difference between the two exponents $(-3.2$ versus -2.9$)$ has a fundamental effect on the wave display clarity. Lastly, we need to point out that it is not straightforward why exponent $\alpha$ varies between different albedo power levels and why it remains roughly consistent along the sectioning curves used to resample the wave detections. The physical mechanism that governs such variability is worth a further investigation. It is probable, as we have argued above, that noise contamination is one cause of the smaller slopes for lower albedo power.

\subsection{Explore the longer-wavelength wave display}

Longer-wavelength ( $>100 \mathrm{~km}$ ) waves are not as visually detectable as the shorter waves because the CIPS orbital strip is not able to embrace many repeats of the ridge and trough of such waves. Chandran et al. (2010) used a wave detection algorithm to yield a peak population of waves at scales of $\sim 250 \mathrm{~km}$ from the cross-track traces. Zhao et al. (2015) yield a peak wavelength at $\sim 400 \mathrm{~km}$ from the along-track traces. Different traces may be the cause of the different peak wavelengths. Based on these studies, gravity waves of all scales are likely widespread in the PMCs. The waves at $<100 \mathrm{~km}$ wavelengths are more visually detectable because their full displays are well captured. But these small-scale waves possess a lower variance level than the larger-scale waves. Using a spectral analysis the larger scales often stand out as the dominant wave events. In addition, wave event counting is not a deterministic procedure. For example, in the current study, the wave detections are forcibly confined within the elliptical regions. Generally speaking, we have to cope with a lot of challenge and uncertainty in the wave tracking study.

Figure 11a presents an example of longer and shorter waves observed together. They are a set of bright and dim cloud bands that suggest wavelengths of $\sim 150-200 \mathrm{~km}$ and shorter waves with wavelengths of $\sim 20-60 \mathrm{~km}$, indicated by the pairs of magenta arrows with solid heads and thin heads, respectively. In this case the CIPS orbital strip achieves pretty satisfying capture of the waves because $\sim 150-200 \mathrm{~km}$ is still a short wavelength relative to the cross-track span $(\sim$ $900 \mathrm{~km}$ ) of the CIPS orbital strip. The albedo map reveals that the longer and shorter waves are nested together and the longer waves appear to have larger amplitudes. The albedo power map in the lower panel shows that the wave power is primarily distributed in the upper-right and lower-left quadrants, which reflects the orientation of the wave ridges and troughs that is perpendicular to this.

The albedo power spectra for this particular case are shown in Fig. 11b, revealing a -3.0 slope over the scale range of $\sim 20-150 \mathrm{~km}$. The correspondence of freq $25=0.34$ and albedo power $=25.0 \times 10^{-12} \mathrm{sr}^{-2}$ makes this case the closest to the medium-albedo-power category shown above, and the -3.0 spectral slope is also close to -2.9 . The spectra are not reliable in either the longwave limit $(>150 \mathrm{~km})$ or the shortwave limit $(<20 \mathrm{~km})$. In the longwave limit the elliptical region does not capture enough repeats of ridge and trough, and as a result the power spectra in this range often readily change when the CWT is applied to a much expanded region $(>400 \mathrm{~km})$ (not shown). In the shortwave limit the measurement noise will contaminate the PMC signals. It is worth mentioning that the $\sim 20-60 \mathrm{~km}$ scale range focused on in this study is for the shortest waves CIPS can resolve due to the $\sim 5 \mathrm{~km}$ spatial resolution and the signal noise lev- 


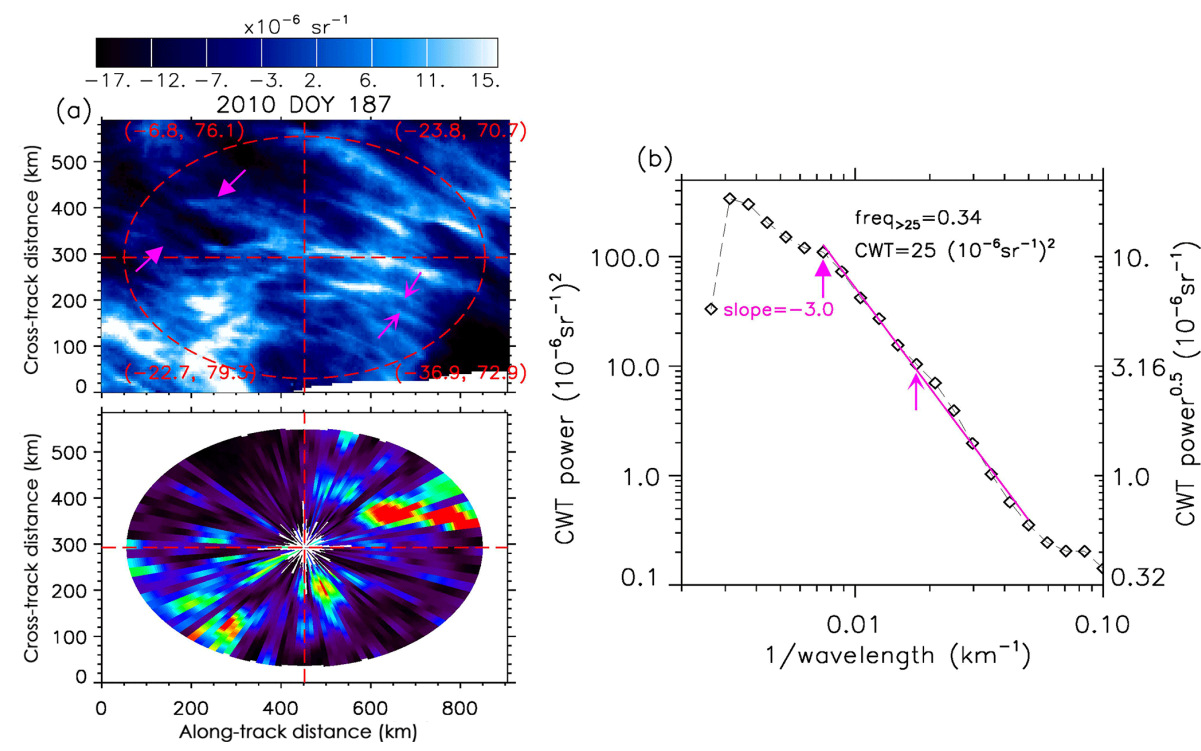

Figure 11. Demonstration of longer and shorter waves nested together. (a) The albedo map and the corresponding albedo power map. The longer waves are roughly at $\sim 150 \mathrm{~km}$ wavelengths (between solid arrows), and the shorter waves are roughly at $\sim 40 \mathrm{~km}$ wavelengths (between thin arrows). (b) The albedo power spectra with an exponent of -3.0 .

els (also see Randall et al., 2017). If $1.0 \times 10^{-6} \mathrm{sr}^{-1}$ is taken as the threshold of the noise level in the CIPS measurements, the wave signatures will be systematically contaminated for the low-albedo-power category based on approximate amplitudes shown in Fig. 6 .

The albedo map and the power spectra in the presumed valid scale range $(\sim 20-150 \mathrm{~km})$ together indicate that longer and shorter waves are nested together with decreasing albedo power. This reminds us of the term "self-similarity", which is used to describe the possible fractal nature of the PMC albedo field with a fractal perimeter dimension of 1.3 (Brinkhoff et al., 2015; von Savigny et al., 2011). Selfsimilarity generally refers to the condition of the small- and large-scale structures resembling each other in morphology. We observed a hint of self-similarity in the gravity wave manifestation described by a $-2.9 \pm 0.3$ law of albedo power, but such an analogy is still preliminary and it requires a further investigation to confirm. It is also worth mentioning that the cascading power spectra from large to small scales (3000$100 \mathrm{~km}$ ) of periodic PMC structures were also reported by Carbary et al. (2000) via analyzing the middle ultraviolet (210-252 nm) images from the Mid-course Space Experiment (MSX) (Carbary et al., 1994). However in their study no structures of $<100 \mathrm{~km}$ wavelengths were detected, and the authors attributed this to the small amplitudes of the shorter-wavelength waves that are not able to rise above the noise level.

\section{Conclusions}

A large ensemble of gravity waves reside in the PMCs, and we aim to extract a universal law that governs the wave display throughout the full set of wave population. More specifically, we examined how wave morphology and the clarity level of display vary throughout the wave population manifested through the PMC albedo data. Higher clarity refers to more distinct exhibition of the features which often correspond to larger variances and a better-organized nature. Later we found that an analytic form of the albedo wavelet power spectra, i.e., $A \times(1 / \text { wavelength })^{\alpha}$, precisely determines the level of display clarity, where the coefficient $A$ and exponent $\alpha$ vary with different sub-groups of wave detections. This form of albedo power spectra is yielded because the gravity wave signatures in the PMCs are mostly quasi-periodic rather than strictly periodic and also because a hierarchy of scale ranges possesses monotonically decreasing power density. The corresponding wavelet power spectra therefore do not exhibit any spectral peaks, especially when spatial averaging of the spectra has been conducted.

A gravity wave tracking algorithm is designed and applied to the PMC albedo data taken by the AIM CIPS instrument to obtain the gravity wave detections throughout the two northern hemispheric summers in 2007 and 2010. The horizontal wavelengths in the range of $\sim 20-60 \mathrm{~km}$ are the focus of the study because they are the most commonly observed and readily captured in the CIPS orbital strips. An individual detection is carried out within an elliptical region of $400 \mathrm{~km}$ along-track and $400 \times 0.65 \mathrm{~km}$ cross-track, and the center of the elliptical region is moved around within any given CIPS Level 2 orbital strip by steps of halved axial lengths in both 
along- and cross-track directions to capture wave signatures at different locations and orientations. For a given location where the elliptical region is placed, a 1-D CWT calculation is carried out in all $360^{\circ}$ directions with $3^{\circ}$ of intervals to obtain the wave power map enclosed in this region. The factor 0.65 in the cross-track direction is empirically chosen owing to the initial intent to better detect the concentric waves in the PMCs. This factor will not qualitatively affect the results of the wave tracking study.

The histograms of the albedo CWT power indicate that a majority of gravity waves of $\sim 20-60 \mathrm{~km}$ wavelengths reside in the lower-albedo-power and lower-background-brightness region, and the number density per bin of the albedo power shows an exponential decay toward high-power values.

The cloud population is split between the dimmer and brighter groups using a defined threshold frequency of the bright-cloud presence to examine the gravity wave manifestation in the dimmer and brighter backgrounds, respectively. Within the elliptical region, if the bright-cloud ( $>$ $25 \times 10^{-6} \mathrm{sr}^{-1}$ ) frequency (freq 25 ) exceeds $2 \%$, then we will call it a brighter background. The threshold $25 \times 10^{-6} \mathrm{sr}^{-1}$ is empirically chosen. Accordingly the dimmer backgrounds refer to the elliptical regions within which freq $_{25}<0.02$. In this study we use both freq 25 and the mean albedo (within the elliptical regions) to describe the background PMC brightness.

The scatterplots of albedo power versus freq 25 and albedo power versus mean albedo (within the elliptical regions) both indicate that statistically albedo power monotonically increases with the PMC background brightness. In this paper we do not pursue what drives such an apparent relationship between the two variables. Rather, we resample the albedo power values to make them conform to a normal distribution based on an analytic form of the albedo power dependence on the background cloud brightness. A sequence of sectioning curves is used to regroup the wave detections. Via the resampling procedure we aim at extracting the law that controls the wave display beyond the apparent dependence of the albedo power on the background cloud brightness. In each resampling bin all brightness levels are included. It is worth noting that the monotonic relationship between the wave amplitude and the background cloud brightness does not seem to reflect the previously discovered driving mechanism between the gravity waves and the local PMC brightness, such as by Jensen and Thomas (1994) and Chandran et al. (2012). These authors suggested that long or short gravity waves eventually reduce the local cloud brightness level.

Sample cases are selected at the two tails and the peak of the normal distribution and at three brightness levels (freq ${ }_{25}=0,0.4$, and 0.8 ) to represent the full set of wave detections. The selections are made following the resampling sectioning curves on the scatterplot and are categorized as possessing high, low, and medium albedo power. As is mentioned above, the resampling procedure is applied to exam- ine the wave display beyond the apparent dependence of the albedo power on the background cloud brightness.

The albedo power spectra over scales $\sim 20-60 \mathrm{~km}$ for the representative cases follow a universal form of $A \times(1 / \text { wavelength })^{\alpha}$, where $\alpha=-3.2,-2.9$, and -2.5 and $A=1.49 \times 10^{-4}, 1.45 \times 10^{-4}$, and $1.42 \times 10^{-4}$ for the high-, medium-, and low-albedo-power categories, respectively. The parameters $A$ and $\alpha$ both take part in determining the overall power magnitude and the decay rate toward the smaller scales. It is worth noting that the three $\alpha$ values will undergo a minor systematic shift when a different form of wavelets (e.g., Mexican hat or Ricker wavelet) is adopted, but they will remain close to -3.0 . In addition, we have argued above that Morlet wavelet is an optimum choice in terms of reflecting both the periodicity and localization of the wave signatures. The overall higher power and the more rapid decay rate both lead to higher clarity of the wave display because the variance level will be higher and meanwhile the leading scale will be more dominant, resulting in a better-organized nature. Each albedo power category possesses a consistent exponent (i.e., $\alpha$ ). As a result, via a simple adjustment of the coefficient $A$, the power spectra between different brightness levels precisely collapse to each other, and therefore a consistent level of display clarity will be achieved. The display clarity degrades substantially from high- to low-albedo-power categories, which seems to suggest that there are widespread and variable diffusive processes at the PMC height. The majority of the detected waves are straight waves or the interference of the straight waves regardless of the background brightness levels. Nevertheless, looking into more details, we found that the wave signatures in the brighter background seem to exhibit mildly turbulentlike features, suggesting that the wave patterns are less linear under this condition.

Exploration of longer-wavelength gravity waves suggests that longer- and shorter-wavelength waves are likely nested together with decreasing albedo power. We may speculate that gravity waves of a hierarchy of scales from $\sim 400-500$ to $\sim 20-60 \mathrm{~km}$ are likely equally widespread and possess exponentially decreasing albedo power density as the wavelength shortens.

Future work includes characterizing the coherency of the wave structures so that the algorithm can work effectively to identify specific wave events and wave morphology. After understanding the power law that governs the overall manifestation of the gravity waves in the PMCs, further identifying individual cases would be vital to eventually understand the mechanism of the gravity wave upward propagation from the source region to the site of display as the algorithm can also be applied to different altitude levels.

Data availability. AIM CIPS data are available to the public at http: //lasp.colorado.edu/aim/download-data-L2.php (LASP, 2018). 
Competing interests. The authors declare that they have no conflict of interest.

Acknowledgements. This work was accomplished at the Center for Atmospheric Sciences, Hampton University, Hampton, Virginia. Funding for the AIM mission was provided by NASA's Small Explorers program under contract NAS5-03132. The project is further supported by NSF funding won in 2017 (award number 1651394). We thank the CIPS retrieval team for their tireless work on the CIPS data retrieval and for the well-maintained and up-to-date status of the online download engine. We appreciate the data archiving team at Hampton University for keeping up the pace of data download onto the local server. We also greatly value the discussion and insights provided by the AIM science team members such as Mike Taylor and Yucheng Zhao during the course of this research work.

Edited by: William Ward

Reviewed by: Christian von Savigny and two anonymous referees

\section{References}

Aumann, H. H., Chahine, M. T., Gautier, C., Goldberg, M. D., Kalnay, E., McMillin, L. M., Revercomb, H., Rosenkranz, P. W., Smith, W. L., Staelin, D. H., Strow L. L., and Susskind J.: AIRS/AMSU/HSB on the Aqua mission: Design, science objective, data products, and processing systems, IEEE T. Geosci. Remote, 41, 253-264, 2003.

Bailey, M. S., Thomas, G. E., Rusch, D. W., Merkel, A. W., Jeppesen, C., Carstens, J. N., Randall, C. E., McClintock, W. E., and Russell III, J. M.: Phase functions of polar mesospheric cloud ice as observed by the CIPS instrument on the AIM satellite, J. Atmos. Sol.-Terr. Phy., 3-4, 373-380, 2009.

Baumgarten, G. and Fritts, D. C.: Quantifying Kelvin-Helmholtz instability dynamics observed in noctilucent clouds: 1 . Methods and observations, J. Geophys. Res.-Atmos., 119, 9324-9337, https://doi.org/10.1002/2014JD021832, 2014.

Brinkhoff, L. A., von Savigny, C., Randall, C. E., and Burrows, J. P.: The fractal perimeter dimension of noctilucent clouds: Sensitivity analysis of the area-perimeter method and results on the seasonal and hemispheric dependence of the fractal dimension, J. Atmos. Sol.-Terr. Phy., 127, 66-72, 2015.

Carbary, J. F., Darlington, E. H., Harris, T. J., McEvaddy, P. J., Mayr, M. J., Peacock, K., and Meng, C. I.: Ultraviolet and visible imaging and spectrographic imaging instrument, Appl. Optics, 3, 4201-4213, 1994.

Carbary, J. F., Morrison, D., and Romick, G. J.: Transpolar structure of polar mesospheric clouds, J. Geophys. Res., 115, 2476324769, 2000.

Chandran, A., Rusch, D. W., Merkel, A. W., Palo, S. E., Thomas, G. E., Taylor, M. J., Bailey, S. M., and Russell III, J. M.: Polar mesospheric cloud structures observed from the cloud imaging and particle size experiment on the Aeronomy of Ice in the Mesosphere spacecraft: Atmospheric gravity waves as drivers for longitudinal variability in polar mesospheric cloud occurrence, J. Geophys. Res., 115, D13102, https://doi.org/10.1029/2009JD013185, 2010.
Chandran, A., Rusch, D. W., Thomas, G. E., Palo, S. E., Baumgarten, G., Jensen, E. J., and Merkel, A. W.: Atmospheric gravity wave effects on polar mesospheric clouds: A comparison of numerical simulations from CARMA 2D with AIM observations, J. Geophys. Res., 117, D20104, https://doi.org/10.1029/2012JD017794, 2012.

Dalin, P., Pertsev, N., Frandsen, S., Hansen, O., Andersen, H., Dubietis, A., and Balciunas, R.: A case study of the evolution of a Kelvin-Helmholtz wave and turbulence in noctilucent clouds, J. Atmos. Sol.-Terr. Phy., 72, 1129-1138, 2010.

DeLand, M. T. and Thomas, G. E.: Updated PMC trends derived from SBUV data, J. Geophys. Res.-Atmos., 120, 2140--2166, https://doi.org/10.1002/2014JD022253, 2015.

Ern, M., Preusse, P., Gille, J. C., Hepplewhite, C. L., Mlynczak, M. G., Russell III, J. M., and Riese, M.: Implications for atmospheric dynamics derived from global observations of gravity wave momentum flux in stratosphere and mesosphere, J. Geophys. Res., 116, D19107, https://doi.org/10.1029/2011JD015821, 2011.

Fogle, B. and Haurwitz, B.: Noctilucent clouds, Space Sci. Rev., 6, 279-340, 1966.

Fritts, D. C. and Alexander, M. J.: Gravity wave dynamics and effects in the middle atmosphere, Rev. Geophys., 41, 1003, https://doi.org/10.1029/2001RG000106, 2003.

Fritts, D. C., Isler, J. R., Thomas, G. E., and Andreassen, Ø.: Wave breaking signatures in noctilucent clouds, Geophys. Res. Lett., 20, 2039-2042, https://doi.org/10.1029/93GL01982, 1993.

Gabor, D.: Theory of communication, J. IEE, 93, 429-459, 1946.

Garcia, R. R. and Solomon, S.: The Effect of Breaking Gravity Waves on the Dynamics and Chemical Composition of the Mesosphere and Lower Thermosphere, J. Geophys. Res., 90, 38503868, 1985.

Gong, J., Yue, J., and Wu, D. L.: Global survey of concentric gravity waves in AIRS images and ECMWF analysis, J. Geophys. Res.-Atmos., 120, 2210-2228, https://doi.org/10.1002/2014JD022527, 2015.

Jensen, E. and Thomas, G. E.: Numerical simulations of the effects of gravity waves on noctilucent clouds, J. Geophys. Res. 99, 3421-3430, 1994.

Laboratory for Atmospheric and Space Physics (LASP): AIM CIPS data, available at: http://lasp.colorado.edu/aim/ download-data-L2.php, last access: January 2018.

Lumpe, J. D., Bailey, S. M., Carstens, J. N., Randall, C. E., Rusch, D. W., Thomas, G. E., Nielsen, K., Jeppesen, C., McClintock, W. E., Merkel, A. W., Riesberg, L., Templeman, B., Baumgarten, G., and Russell III, J. M.: Retrieval of polar mesospheric cloud properties from CIPS: Algorithm description, error analysis and cloud detection sensitivity, J. Atmos. Sol.-Terr. Phy., 104, 167196, https://doi.org/10.1016/j.jastp.2013.06.007, 2013.

Matsuda, T. S., Nakamura, T., Ejiri, M. K., Tsutsumi, M., and Shiokawa, K.: New statistical analysis of the horizontal phase velocity distribution of gravity waves observed by airglow imaging, J. Geophys. Res.-Atmos., 119, 9707-9718, https://doi.org/10.1002/2014JD021543, 2014.

McClintock, W. E., Rusch, D. W., Thomas, G. E., Merkel, A. W., Lankton, M. R., Drake, V. A., Bailey, S. M., and Russell III, J. M.: The cloud imaging and particle size experiment on the Aeronomy of Ice in the Mesosphere mission: Instrument concept, design, calibration, and on- 
orbit performance, J. Atmos. Sol.-Terr. Phy., 71, 340-355, https://doi.org/10.1016/j.jastp.2008.10.011, 2009.

Merkel, W. A., Rusch, D. W., Palo, S. E., Russell III, J. M., and Bailey, S. M.: Mesospheric planetary wave activity inferred from AIM-CIPS and TIMED-SABER for the northern summer 2007 PMC season, J. Atmos. Sol.-Terr. Phy., 71, 381-391, https://doi.org/10.1016/j.jastp.2008.12.001, 2009.

Miller, A. D., Fritts, D. C., Chapman, D., Jones, G., Limon, M., Araujo, D., Didier, J., Hillbrand, S., Kjellstrand, C. B., Korotkov, A., Tucker, G., Vinokurov, Y., Wan, K., and Wang, L.: Stratospheric imaging of polar mesospheric clouds: A new window on small-scale atmospheric dynamics, Geophys. Res. Lett., 42, 6058-6065, https://doi.org/10.1002/2015GL064758, 2015.

Randall, C. E., Carstens, J., France, J. A., Harvey, V. L., Hoffmann, L., Bailey, S. M., Alexander, M. J., Lumpe, J. D., Yue, J., Thurairajah, B., Siskind, D. E., Zhao, Y., Taylor, M. J., and Russell III, J. M.: New AIM/CIPS global observations of gravity waves near 50-55 km, Geophys. Res. Lett., 44, 7044-7052, https://doi.org/10.1002/2017GL073943, 2017.

Russell III, J. M., Bailey, S. M., Gordley, L. L., Rusch, D. W., Horányi, M., Hervig, M. E., Thomas, G. E., Randall, C. E., Siskind, D. E., Stevens, M. H., Summers, M. E., Taylor, M. J., Englert, C. R., Espy, P. J., McClintock, W. E., and Merkel, A. W.: Aeronomy of Ice in the Mesosphere (AIM) mission: Overview and early science results, J. Atmos. Sol.-Terr. Phy., 71, 289-299, https://doi.org/10.1016/j.jastp.2008.08.011, 2009.

Taylor, M. J. and Edwards, R.: Observations of Short Period Mesospheric Wave Patterns: In Situ or Tropospheric Wave Generation, Geophys. Res. Lett., 18, 1337-1340, 1991.

Taylor, M. J., Pautet, P.-D., Zhao, Y., Randall, C. E., Lumpe, J., Bailey, S. M., Carstens, J., Nielsen, K., Russell III, J. M., and Stegman, J.: High-latitude gravity wave measurements in noctilucent clouds and polar mesospheric clouds, in: Aeronomy of the Earth's Atmosphere and Ionosphere, IAGA Spec. Sopron Book Ser., vol. 2, edited by: Abdu, M. A. and Pancheva, D., Part 1, 93-105, Springer, the Netherlands, https://doi.org/10.1007/978-94-007-0326-1_7, 2011.
Thurairajah, B., Bailey, S. M., Nielsen, K., Randall, C. E., Lumpe, J. D., Taylor, M. J., and Russell III, J. M.: Morphology of polar mesospheric clouds as seen from space, J. Atmos. Sol.-Terr. Phy., 104, 234-243, https://doi.org/10.1016/j.jastp.2012.09.009, 2013.

Vadas, S. L., Yue, J., She, C.-Y., Stamus, P. A., and Liu, A. Z.: A model study of the effects of winds on concentric rings of gravity waves from a convective plume near Fort Collins on 11 May 2004, J. Geophys. Res., 114, D06103, https://doi.org/10.1029/2008JD010753, 2009.

von Savigny, C., Brinkhoff, L. A., Bailey, S. M., Randall, C. E., and Russell III, J. M.: First determination of the fractal perimeter dimension of noctilucent clouds, Geophys. Res. Lett., 38, L02806, https://doi.org/10.1029/2010GL045834, 2011.

Wachter, P., Schmidt, C., Wuest, S., and Bittner, M.: Spatial gravity wave characteristics obtained from multiple $\mathrm{OH}(3-1)$ airglow temperature time series, J. Atmos. Sol.-Terr. Phy., 135, 192-201, 2015.

Yue, J., Vadas, S. L., She, C.-Y., Nakamura, T., Reising, S. C., Liu, H.-L., Stamus, P., Krueger, D. A., Lyons, W., and $\mathrm{Li}, \mathrm{T}$.: Concentric gravity waves in the mesosphere generated by deep convective plumes in the lower atmosphere near Fort Collins, Colorado, J. Geophys. Res., 114, D06104, https://doi.org/10.1029/2008JD011244, 2009.

Yue, J., Thurairajah, B., Hoffmann, L., Alexander, J., Chandran, A., Taylor, M. J., Russell III, J. M., Randall, C. E., and Bailey, S. M.: Concentric gravity waves in polar mesospheric clouds from the Cloud Imaging and Particle Size experiment, J. Geophys. Res.-Atmos., 119, 5115-5127, https://doi.org/10.1002/2013JD021385, 2014.

Zhao, Y., Taylor, M. J., Randall, C. E., Lumpe, J. D., Siskind, D. E., Bailey, S. M., and Russell III, J. M.: Investigating seasonal gravity wave activity in the summer polar mesosphere, J. Atmos. Sol.-Terr. Phy., 127, 289-299, https://doi.org/10.1016/j.jastp.2015.03.008, 2015. 